\title{
Breeding bird communities in an area of the Northern Apennines (Piedmont, NW Italy)
}

\author{
Franco Carpegna ${ }^{1}$, Giovanni Soldato ${ }^{2}$, Roberto Toffoli ${ }^{3 *}$
}

\begin{abstract}
During the Spring of 2011, we studied the bird community in an area of Val Borbera, in the province of Alessandria (NW Italy). In the study area, situated at an altitude between 655 and 1700 $\mathrm{m}$ a.s.1., we conducted 110 points count ten minutes each in four microhabitats (agricultural areas, shrubs, forests, and prairies).

In total, we surveyed 72 species, of which 51 were passerine and 21 non passerine. The most abundant species were Sylvia atricapilla, Phylloscopus collybita, Apus apus, and Turdus merula. In the agricultural areas, we detected a total of 50 species (Sylvia atricapilla, Parus major, Turdus merula were the most abundant). In the shrubs, we detected 30 species (Sylvia atricapilla, Parus major, Fringilla coelebs, Phylloscopus collybita, Turdus merula, Erithacus rubecula were the most abundant). In the forest areas, we found 45 species (Sylvia atricapilla, Parus major, Fringilla coelebs, Phylloscopus collybita, Turdus merula, Erithacus rubecula were the most abundant), and in the prairies, we detected 48 species (Alauda arvensis, Anthus campestris, Sylvia atricapilla, Turdus merula, Anthus trivialis, Sylvia communis were the most abundant).

Compared to the other macro habitats, the agricultural areas have a significantly high abundance and richness in species, which highlights the importance of the agricultural mosaics in the Piedmont and mountain areas. The data which has been collected so far confirms the important role of this area, given the presence of some species which are rare at a regional scale.
\end{abstract}

Key words: bird communities, richness, abundance, dominance, diversity, Northern Apennines.

Riassunto - La comunità di uccelli nidificanti in un'area dell'Appennino settentrionale (Piemonte, Italia NO).

Durante la primavera 2011 abbiamo studiato la comunità di uccelli di un'area della Val Borbera (provincia di Alessandria, Italia Nord occidentale). Nell'area di studio, ad un'altitudine compresa tra 655 e 1700 $\mathrm{m}$ s.l.m. abbiamo eseguito 110 punti d'ascolto della durata di 10 minuti, in quattro macro-habitat (ambienti agrari, arbusteti, ambienti forestali e praterie).

${ }^{1}$ Via San Pio XXIII 27, 10126 Torino, Italia.

Email: carpifc@libero.it

${ }^{2}$ Via Ormea 130, 10126 Torino, Italia.

Email: giovanni.soldato@gmail.com

${ }^{3}$ Via P. Viada 3/B, 12018, Roccavione (CN), Italia.

* Corresponding author: rtoffoli@iol.it

(C) 2018 Franco Carpegna, Giovanni Soldato, Roberto Toffoli

Received: 10 January 2018

Accepted for publication: 22 January 2019

Online publication: 18 June 2019
In totale sono state rilevate 72 specie tra le quali 21 non Passeriformi e 51 Passeriformi. Le specie più abbondanti sono state: capinera, fringuello, rondone e merlo.

Negli ambienti agrari abbiamo rilevato un totale di 50 specie (capinera, cinciallegra, merlo le più abbondanti), negli arbusteti 30 specie (capinera, cinciallegra, fringuello, luì piccolo, merlo, pettirosso), negli ambienti forestali 45 specie (capinera, cinciallegra, fringuello, luì piccolo, merlo, pettirosso) e nelle praterie 48 specie (allodola, calandro, capinera, merlo, prispolone, sterpazzola).

La ricchezza di specie e l'abbondanza è risultata significativamente più elevata negli ambienti agrari rispetto agli altri macro habitat, evidenziando l'importanza dei mosaici agrari nelle aree pedemontane e montane. I dati raccolti confermano il ruolo di quest'area per la presenza di alcune specie poco diffuse a scala regionale.

Parole chiave: comunità ornitica, ricchezza specifica, abbondanza, dominanza, diversità, Appennino settentrionale.

\section{INTRODUCTION}

Piedmont's sector of the Northern Apennines is, to this day, still scarcely known from an ornithological point of view. Excluding the information on a regional scale (Mingozzi et al., 1988; Aimassi \& Reteuna, 2007), ornithological research is scarce and exclusively regard data lists (Silvano et al., 1988) or nesting communities in single protected areas (Toffoli et al., 2003). The limited available information highlights the presence of diversified communities in the Apennines sector, in which you can observe the presence of species with a prevalently Alpine distribution, despite the not particularly elevated heights, and of principally Apennines and Mediterranean species.

The purpose of this study is to contribute to the knowledge on the avifauna of this mountain area in order to obtain information about the distribution and local ecology of the different species. Furthermore, another intent is to provide knowledge in order to evaluate the state of conservation in the habitats of a mountain sector subjected to radical changes in soil cover due to the abandonment of agricultural practices (Assini et al., 2014).

\section{AREA OF STUDY}

Our study has been conducted in an area of 9.874 hectares of the Piedmont's sector of the Apennines (province of Alessandria, North-West Italy) between the valleys of Borbera and Curone, along the ridge between Mount Giarolo and Mount Ebro, at an altitude between $655 \mathrm{~m}$ and $1700 \mathrm{~m}$ 
(Fig. 1). The area of study also includes a part of the SPA IT1180025 Ridge Mount Ebro and Mount Chiappo.

From an environmental point of view, the area is comprised of a mosaic of open space habitats, shrubs, and forest vegetation. According to the Forest Territorial Plan of the Piedmont region (Regione Piemonte, 2009), open space areas are mainly comprised of prairies $(10 \%$ of the study area), meadow pastures (8\%), and arable land (4\%). The woods are comprised primarily of mixed deciduous forest (21\%), beeches (19\%), oak woods of Quercus pubescens and Quercus cerris (16\%), and chestnut woods $(6 \%)$. The remaining land covers are comprised of brushwoods, invading thickets, abandoned crop lands, and small inhabited areas.

\section{MATERIALS AND METHODS}

The surveys conducted from the $17^{\text {th }}$ of May to the $28^{\text {th }}$ of June 2011 in 110-points counts are ten minutes long (Blondel et al., 1970; Bibby et al., 2000; Fornasari et al., 2010). In total, 110 casual point counts where made/ established between the $17^{\text {th }}$ of May and the $28^{\text {th }}$ of June 2011. These recordings where made at a distance of 500 meters and in favourable meteorological conditions, in absence of winds and precipitations, between dawn and 11:00 AM. For every point count, all the visible and heard individuals were counted and subdivided within and above 100 meters.
The listening points have been subdivided in four environmental categories of ornithological interest gathered from the cartography of the Forest Territorial Plan of the Piedmont region (Regione Piemonte, 2009): i) Agricultural areas 21 points; ii) Environments in evolution (shrubs) 11 points; iii) Forest areas 45 points; iv) Prairies 33 points.

The forest environments have been subdivided into further sub-categories gathered from the Forest Territorial Plan: chestnut woods, beech woods, oak woods, mixed deciduous woods, and reforested areas. Agricultural areas and prairies have been subdivided into the following subcategories based on their presence within 300 meters from the point count of the edge of woods, shrub or uncultivated areas, hedges or other elements of the landscape taken from the Forest Territorial Plan: heterogeneous agricultural areas, non-heterogeneous agricultural areas, heterogeneous prairies, and non-heterogeneous prairies.

The nesting ornithological community has been characterised calculating the following parameters:

- richness (S): total number of surveyed species;

- mean richness (mean $\mathrm{S}$ ): mean number of surveyed species per point/site;

- abundance (A): mean number of individuals per point/ site;

- dominance (pi): where pi is the relative frequency of each species; species with $\mathrm{pi} \geq 0.05$ and $\mathrm{pi} \geq 0.02$ are considered respectively dominant and sub dominant (Turcek, 1956);

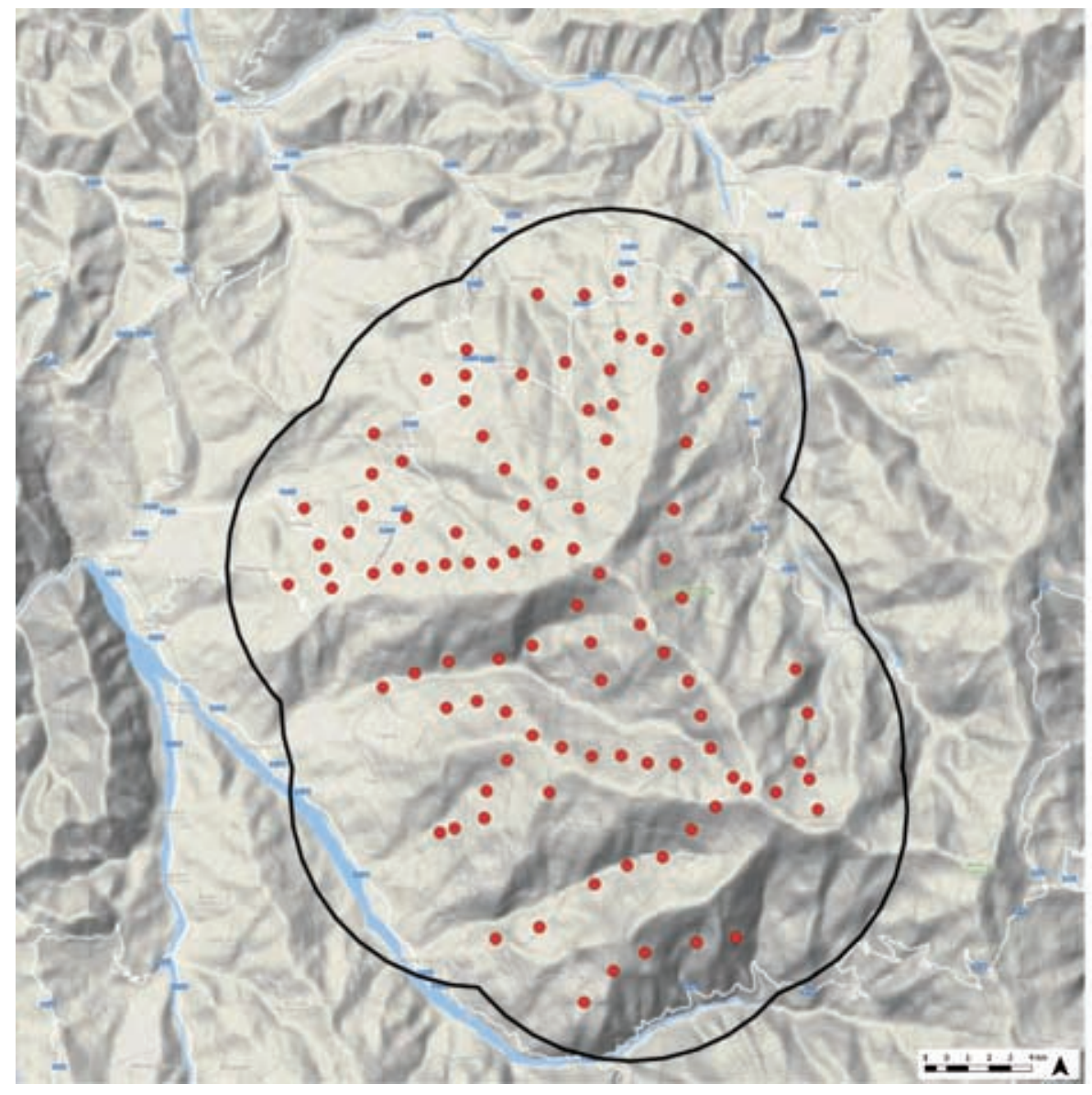

Fig. 1 - Study area. / Area di studio.

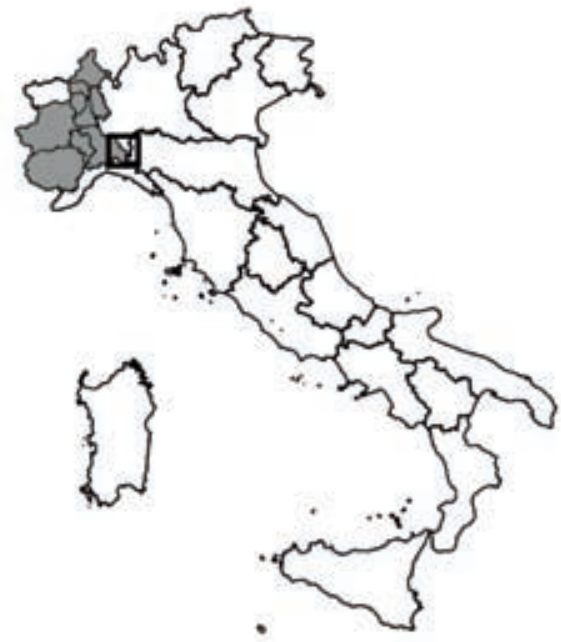

Study area

Point counts 
- Shannon's Index $(H)$ : Shannon's Index $\left(H^{\prime}=-\sum\right.$ pi log pi) (Shannon \& Weaver, 1963);

- Evenness (J): $\mathrm{J}=\mathrm{H} / \operatorname{lnS}$ (Lloyd \& Ghelardi, 1964; Pielou, 1966);

- Percentage of non-Passeriformes (Ferry \& Frochot, 1970).

- Percentage of declining species: categories Critically Endangered (CR), Endangered (EN), Vulnerable (VU), Near-Threatened (NT) of the National IUCN Red List (Peronace et al., 2012).

For the analysis of the parameters of the communities and the relative frequencies of the species surveyed in the study area, we used the data collected at every listening point, both within and outside of the 100 meter range from the observer. In the analysis of the bird communities within the four environmental typologies considered separately, we considered only the data surveyed within the $100 \mathrm{~m}$ range. As for the environmental sub categories of the forest areas, we considered as parameters of the communities only the species richness and the mean number of species per site/point, given the limited number of survey station/sites.

Given that the collected data were not normally distributed - test of Kolmogorov-Smirnov - we used non parametric tests for the statistical analysis; the percentages of non passerines have been analysed through the $\chi 2$ test, while the differences in species richness and abundance between the four habitats have been evaluated with the Kruskal-Wallis test, and we adopted the Dunn test for multiple confrontations between habitats. This approach has also been used to confront the mean richness within the subcategories in the forest areas. The differences in dominion of the surveyed species in the four habitats have been tested with Friedman's test, and we adopted the Dunn test for multiple confrontations between habitats. The community parameters have been calculated using the PAST software (Hammer et al., 2001), while the statistical analysis was conducted with PRISM 5.

In the tables, species where listed by EURING code.

\section{RESULTS}

We observed 72 species, 21 of which non passerine (29.2\%) and 51 passerine (70.8\%). The dominant species are, in decreasing order, Sylvia atricapilla, Phylloscopus collybita, Apus apus, and Turdus merula; the sub dominant species are seven. The most frequent species, which were spotted in over $50 \%$ on the sites are, in decreasing order, Turdus merula, Sylvia atricapilla, Fringilla coelebs, and Phylloscopus collybita (Tab. 1). In Tab. 2, the community parameters calculated in the study area are reported.

In the four environmental categories taken into consideration, we surveyed 64 species (within a 100 meter range), the abundance and dominance of which are reported in Tab. 3.

Tab.1 - List of species detected with the percentage frequency and dominance (in bold underlined on dominant species pi $>0.05$ Turcek, 1956). Threat categories from National IUCN Red List (Peronace et al., 2012). / Elenco delle specie rilevate con relative frequenza percentuale e dominanza (in grassetto sottolineato le specie dominant pi $>0,05$ Turcek, 1956). Categorie di minaccia tratte dalla Lista Rossa IUCN nazionale (Peronace et al., 2012).

\begin{tabular}{|l|c|c|c|c|c|c|c|}
\hline Species & $\begin{array}{c}\text { N. } \\
\text { points }\end{array}$ & $\begin{array}{c}\text { Frequence } \\
\text { \% }\end{array}$ & Individuals & $\begin{array}{c}\text { Mean individuals/ } \\
\text { points }\end{array}$ & SD & pi & $\begin{array}{c}\text { National } \\
\text { Red List }\end{array}$ \\
\hline Pernis apivorus & 9 & 8.18 & 10 & 0.09 & 0.35 & 0.01 & LC \\
\hline Circaetus gallicus & 3 & 2.73 & 2 & 0.02 & 0.13 & 0.00 & VU \\
\hline Accipiter gentilis & 1 & 0.91 & 1 & 0.01 & 0.10 & 0.00 & LC \\
\hline Accipiter nisus & 5 & 4.55 & 5 & 0.05 & 0.21 & 0.00 & LC \\
\hline Buteo buteo & 9 & 8.18 & 12 & 0.11 & 0.39 & 0.01 & LC \\
\hline Aquila chrysaetos & 1 & 0.91 & 1 & 0.01 & 0.10 & 0.00 & NT \\
\hline Falco tinnunculus & 3 & 2.73 & 2 & 0.02 & 0.13 & 0.00 & LC \\
\hline Falco subbuteo & 2 & 1.82 & 4 & 0.04 & 0.30 & 0.00 & LC \\
\hline Alectoris rufa & 3 & 2.73 & 4 & 0.04 & 0.23 & 0.00 & DD \\
\hline Coturnix coturnix & 1 & 0.91 & 1 & 0.01 & 0.10 & 0.00 & DD \\
\hline Phasianus colchicus & 4 & 3.64 & 3 & 0.03 & 0.16 & 0.00 & NA \\
\hline Columba palumbus & 7 & 6.36 & 6 & 0.05 & 0.23 & 0.00 & LC \\
\hline Streptopelia decaocto & 4 & 3.64 & 7 & 0.06 & 0.43 & 0.00 & LC \\
\hline Streptopelia turtur & 12 & 10.91 & 18 & 0.16 & 0.57 & 0.01 & LC \\
\hline Cuculus canorus & 48 & 43.64 & 50 & 0.45 & 0.55 & 0.03 & LC \\
\hline Otus scops & 1 & 0.91 & 1 & 0.01 & 0.10 & 0.00 & LC \\
\hline Strix aluco & 1 & 0.91 & 1 & 0.01 & 0.10 & 0.00 & LC \\
\hline
\end{tabular}




\begin{tabular}{|c|c|c|c|c|c|c|c|}
\hline Species & $\begin{array}{c}\text { N. } \\
\text { points }\end{array}$ & $\begin{array}{c}\text { Frequence } \\
\%\end{array}$ & Individuals & $\begin{array}{l}\text { Mean individuals/ } \\
\text { points }\end{array}$ & SD & pi & $\begin{array}{l}\text { National } \\
\text { Red List } \\
\end{array}$ \\
\hline Apus apus & 14 & 12.73 & 125 & 1.14 & 4.99 & 0.08 & $\mathrm{LC}$ \\
\hline Picus viridis & 4 & 3.64 & 3 & 0.03 & 0.16 & 0.00 & $\mathrm{LC}$ \\
\hline Dendrocopos major & 11 & 10.00 & 10 & 0.09 & 0.29 & 0.01 & $\mathrm{LC}$ \\
\hline Dendrocopos minor & 1 & 0.91 & 1 & 0.01 & 0.10 & 0.00 & $\mathrm{LC}$ \\
\hline Lullula arborea & 20 & 18.18 & 22 & 0.20 & 0.47 & 0.01 & $\mathrm{LC}$ \\
\hline Alauda arvensis & 23 & 20.91 & 57 & 0.52 & 1.21 & 0.04 & VU \\
\hline Ptyonoprogne rupestris & 1 & 0.91 & 2 & 0.02 & 0.19 & 0.00 & $\mathrm{LC}$ \\
\hline Hirundo rustica & 12 & 10.91 & 22 & 0.20 & 0.71 & 0.01 & NT \\
\hline Delichon urbicum & 1 & 0.91 & 2 & 0.02 & 0.19 & 0.00 & NT \\
\hline Anthus campestris & 13 & 11.82 & 19 & 0.17 & 0.52 & 0.01 & $\mathrm{LC}$ \\
\hline Anthus trivialis & 32 & 29.09 & 45 & 0.41 & 0.73 & 0.03 & VU \\
\hline Anthus spinoletta & 2 & 1.82 & 2 & 0.02 & 0.13 & 0.00 & $\mathrm{LC}$ \\
\hline Motacilla cinerea & 1 & 0.91 & 1 & 0.01 & 0.10 & 0.00 & $\mathrm{LC}$ \\
\hline Motacilla alba & 10 & 9.09 & 13 & 0.12 & 0.44 & 0.01 & $\mathrm{LC}$ \\
\hline Troglodytes troglodytes & 12 & 10.91 & 13 & 0.12 & 0.35 & 0.01 & $\mathrm{LC}$ \\
\hline Erithacus rubecula & 48 & 43.64 & 71 & 0.65 & 0.84 & 0.05 & $\mathrm{LC}$ \\
\hline Luscinia megarhynchos & 9 & 8.18 & 9 & 0.08 & 0.31 & 0.01 & $\mathrm{LC}$ \\
\hline Phoenicurus ochruros & 10 & 9.09 & 9 & 0.08 & 0.28 & 0.01 & $\mathrm{LC}$ \\
\hline Phoenicurus phoenicurus & 16 & 14.55 & 20 & 0.18 & 0.49 & 0.01 & $\mathrm{LC}$ \\
\hline Saxicola torquatus & 9 & 8.18 & 9 & 0.08 & 0.31 & 0.01 & VU \\
\hline Turdus merula & 77 & 70.00 & 117 & 1.06 & 0.92 & 0.08 & $\mathrm{LC}$ \\
\hline Turdus philomelos & 21 & 19.09 & 25 & 0.23 & 0.50 & 0.02 & $\mathrm{LC}$ \\
\hline Turdus viscivorus & 7 & 6.36 & 7 & 0.06 & 0.25 & 0.00 & $\mathrm{LC}$ \\
\hline Sylvia subalpina & 8 & 7.27 & 7 & 0.06 & 0.25 & 0.00 & $\mathrm{LC}$ \\
\hline Sylvia communis & 22 & 20.00 & 29 & 0.26 & 0.62 & 0.02 & $\mathrm{LC}$ \\
\hline Sylvia atricapilla & 74 & 67.27 & 151 & 1.37 & 1.23 & 0.10 & $\mathrm{LC}$ \\
\hline Phylloscopus bonelli & 18 & 16.36 & 19 & 0.17 & 0.43 & 0.01 & $\mathrm{LC}$ \\
\hline Phylloscopus collybita & 55 & 50.00 & 66 & 0.60 & 0.68 & 0.04 & $\mathrm{LC}$ \\
\hline Regulus ignicapilla & 6 & 5.45 & 6 & 0.05 & 0.23 & 0.00 & $\mathrm{LC}$ \\
\hline Muscicapa striata & 6 & 5.45 & 7 & 0.06 & 0.28 & 0.00 & $\mathrm{LC}$ \\
\hline Aegithalos caudatus & 15 & 13.64 & 25 & 0.23 & 0.73 & 0.02 & $\mathrm{LC}$ \\
\hline Poecile palustris & 18 & 16.36 & 20 & 0.18 & 0.43 & 0.01 & $\mathrm{LC}$ \\
\hline Lophophanes cristatus & 8 & 7.27 & 9 & 0.08 & 0.31 & 0.01 & $\mathrm{LC}$ \\
\hline Periparus ater & 13 & 11.82 & 17 & 0.15 & 0.45 & 0.01 & $\mathrm{LC}$ \\
\hline Cyanistes caeruleus & 38 & 34.55 & 52 & 0.47 & 0.77 & 0.03 & $\mathrm{LC}$ \\
\hline Parus major & 53 & 48.18 & 62 & 0.56 & 0.66 & 0.04 & $\mathrm{LC}$ \\
\hline Sitta europaea & 17 & 15.45 & 18 & 0.16 & 0.42 & 0.01 & $\mathrm{LC}$ \\
\hline Certhia familiaris & 11 & 10.00 & 12 & 0.11 & 0.34 & 0.01 & $\mathrm{LC}$ \\
\hline Lanius collurio & 10 & 9.09 & 13 & 0.12 & 0.40 & 0.01 & VU \\
\hline Garrulus glandarius & 25 & 22.73 & 30 & 0.27 & 0.56 & 0.02 & $\mathrm{LC}$ \\
\hline Pica pica & 3 & 2.73 & 4 & 0.04 & 0.23 & 0.00 & $\mathrm{LC}$ \\
\hline Corvus cornix & 8 & 7.27 & 13 & 0.12 & 0.52 & 0.01 & $\mathrm{LC}$ \\
\hline Sturnus vulgaris & 1 & 0.91 & 2 & 0.02 & 0.19 & 0.00 & $\mathrm{LC}$ \\
\hline Passer italiae & 5 & 4.55 & 24 & 0.22 & 1.17 & 0.02 & VU \\
\hline Passer montanus & 1 & 0.91 & 4 & 0.04 & 0.38 & 0.00 & VU \\
\hline
\end{tabular}




\begin{tabular}{|l|c|c|c|c|c|c|c|}
\hline Species & $\begin{array}{c}\text { N. } \\
\text { points }\end{array}$ & $\begin{array}{c}\text { Frequence } \\
\mathbf{\%}\end{array}$ & Individuals & $\begin{array}{c}\text { Mean individuals/ } \\
\text { points }\end{array}$ & SD & pi & $\begin{array}{c}\text { National } \\
\text { Red List }\end{array}$ \\
\hline Fringilla coelebs & 68 & 61.82 & 133 & 1.21 & 1.20 & $\mathbf{0 . 0 9}$ & LC \\
\hline Serinus serinus & 12 & 10.91 & 18 & 0.16 & 0.53 & 0.01 & LC \\
\hline Carduelis chloris & 12 & 10.91 & 22 & 0.20 & 0.65 & 0.01 & NT \\
\hline Carduelis carduelis & 11 & 10.00 & 12 & 0.11 & 0.37 & 0.01 & NT \\
\hline Carduelis cannabina & 6 & 5.45 & 10 & 0.09 & 0.42 & 0.01 & NT \\
\hline Pyrrhula pyrrhula & 4 & 3.64 & 5 & 0.05 & 0.25 & 0.00 & VU \\
\hline Emberiza citrinella & 5 & 4.55 & 6 & 0.05 & 0.23 & 0.00 & LC \\
\hline Emberiza cirlus & 18 & 16.36 & 20 & 0.18 & 0.45 & 0.01 & LC \\
\hline Emberiza cia & 5 & 4.55 & 6 & 0.05 & 0.27 & 0.00 & LC \\
\hline Emberiza calandra & 2 & 1.82 & 3 & 0.03 & 0.21 & 0.00 & LC \\
\hline
\end{tabular}

Tab.2 - Breeding bird community parameters: S=Richness, Smean=mean Richness per point count, $\mathrm{A}=$ Abundance, $\mathrm{H}=$ Diversity, $\mathrm{J}=$ Evenness, Non Pass. $=\%$ non-Passeriformes, $\mathrm{N}$ dom. $=$ number of dominant species (see Methods), Ts $=\%$ Threatened species (see Methods). / Parametri della comunità ornitica nidificante: $\mathrm{S}=$ Ricchezza, Smean=Ricchezza media per punto; $\mathrm{A}=$ Abbondanza, $\mathrm{H}=$ Diversità, $\mathrm{J}=$ Equiripartizione, Non Pass. $=\%$ non-Passeriformes, N. dom. $=$ numero di specie dominanti (vedi Metodi), $\mathrm{Ts}=\%$ percentuale specie minacciate (vedi Metodi)

\begin{tabular}{|l|c|c|c|c|c|c|c|c|c|}
\hline & Point counts & S & Smean & A & H & J & Non Pass. & N dom. & Ts \\
\hline Study area & 110 & 72 & 8.1 & 14.2 & 3.58 & 0.83 & 29.2 & 4 & 12.7 \\
\hline
\end{tabular}

Tab.3 - Mean number of individuals per point count $( \pm$ SD) and dominance values in each habitat type (in bold underlined on dominant species pi $>0.05$ Turcek, 1956). / Numero medio di individui per punto ( \pm DS) e valori di dominanza per ogni categoria ambientale (in grassetto sottolineato le specie dominanti pi $>0,05$ Turcek, 1956)

\begin{tabular}{|l|c|c|c|c|c|c|c|c|c|c|c|c|}
\hline & \multicolumn{3}{|c|}{ Agricultural areas } & \multicolumn{4}{|c|}{ Shrubs } & \multicolumn{3}{c|}{ Forest areas } & \multicolumn{3}{c|}{ Prairies } \\
\hline Species & Mean & SD & Pi & Mean & SD & pi & Mean & SD & Pi & Mean & SD & pi \\
\hline Accipiter nisus & 0.00 & 0.00 & 0.00 & 0.09 & 0.30 & 0.01 & 0.00 & 0.00 & 0.00 & 0.03 & 0.17 & 0.00 \\
\hline Falco tinnunculus & 0.05 & 0.22 & 0.00 & 0.00 & 0.00 & 0.00 & 0.00 & 0.00 & 0.00 & 0.03 & 0.17 & 0.00 \\
\hline Falco Subbuteo & 0.00 & 0.00 & 0.00 & 0.00 & 0.00 & 0.00 & 0.00 & 0.00 & 0.00 & 0.03 & 0.17 & 0.00 \\
\hline Alectoris rufa & 0.05 & 0.22 & 0.00 & 0.00 & 0.00 & 0.00 & 0.00 & 0.00 & 0.00 & 0.09 & 0.38 & 0.01 \\
\hline Coturnix coturnix & 0.00 & 0.00 & 0.00 & 0.00 & 0.00 & 0.00 & 0.00 & 0.00 & 0.00 & 0.03 & 0.17 & 0.00 \\
\hline Phasianus colchicus & 0.05 & 0.22 & 0.00 & 0.00 & 0.00 & 0.00 & 0.02 & 0.15 & 0.00 & 0.00 & 0.00 & 0.00 \\
\hline Columba palumbus & 0.05 & 0.22 & 0.00 & 0.18 & 0.40 & 0.02 & 0.02 & 0.15 & 0.00 & 0.03 & 0.17 & 0.00 \\
\hline Streptopelia decaocto & 0.24 & 0.89 & 0.02 & 0.00 & 0.00 & 0.00 & 0.04 & 0.30 & 0.00 & 0.00 & 0.00 & 0.00 \\
\hline Streptopelia turtur & 0.29 & 0.56 & 0.02 & 0.00 & 0.00 & 0.00 & 0.18 & 0.53 & 0.02 & 0.00 & 0.00 & 0.00 \\
\hline Cuculus canorus & 0.24 & 0.44 & 0.02 & 0.00 & 0.00 & 0.00 & 0.11 & 0.32 & 0.01 & 0.15 & 0.36 & 0.02 \\
\hline Apus apus & 0.67 & 2.22 & 0.04 & 0.00 & 0.00 & 0.00 & 0.00 & 0.00 & 0.00 & 0.30 & 1.74 & 0.03 \\
\hline Picus viridis & 0.10 & 0.30 & 0.01 & 0.09 & 0.30 & 0.01 & 0.00 & 0.00 & 0.00 & 0.00 & 0.00 & 0.00 \\
\hline Dendrocopos major & 0.10 & 0.30 & 0.01 & 0.00 & 0.00 & 0.00 & 0.16 & 0.37 & 0.02 & 0.03 & 0.17 & 0.00 \\
\hline Dendrocopos minor & 0.00 & 0.00 & 0.00 & 0.09 & 0.30 & 0.01 & 0.00 & 0.00 & 0.00 & 0.00 & 0.00 & 0.00 \\
\hline Lullula arborea & 0.10 & 0.30 & 0.01 & 0.00 & 0.00 & 0.00 & 0.07 & 0.33 & 0.01 & 0.39 & 0.56 & 0.04 \\
\hline Alauda arvensis & 0.00 & 0.00 & 0.00 & 0.00 & 0.00 & 0.00 & 0.07 & 0.33 & 0.01 & 1.18 & 1.24 & $\mathbf{0 . 1 2}$ \\
\hline Hirundo rustica & 0.43 & 0.81 & 0.03 & 0.18 & 0.40 & 0.02 & 0.00 & 0.00 & 0.00 & 0.03 & 0.17 & 0.00 \\
\hline Delichon urbicum & 0.10 & 0.44 & 0.01 & 0.00 & 0.00 & 0.00 & 0.00 & 0.00 & 0.00 & 0.00 & 0.00 & 0.00 \\
\hline Anthus campestris & 0.00 & 0.00 & 0.00 & 0.00 & 0.00 & 0.00 & 0.00 & 0.00 & 0.00 & 0.58 & 0.83 & $\mathbf{0 . 0 6}$ \\
\hline Anthus trivialis & 0.00 & 0.00 & 0.00 & 0.09 & 0.30 & 0.01 & 0.07 & 0.25 & 0.01 & 1.06 & 0.61 & $\mathbf{0 . 1 1}$ \\
\hline
\end{tabular}




\begin{tabular}{|c|c|c|c|c|c|c|c|c|c|c|c|c|}
\hline \multirow[b]{2}{*}{ Species } & \multicolumn{3}{|c|}{ Agricultural areas } & \multicolumn{3}{|c|}{ Shrubs } & \multicolumn{3}{|c|}{ Forest areas } & \multicolumn{3}{|c|}{ Prairies } \\
\hline & Mean & SD & $\mathrm{Pi}$ & Mean & SD & pi & Mean & SD & $\mathrm{Pi}$ & Mean & SD & pi \\
\hline Anthus spinoletta & 0.00 & 0.00 & 0.00 & 0.00 & 0.00 & 0.00 & 0.00 & 0.00 & 0.00 & 0.06 & 0.24 & 0.01 \\
\hline Motacilla cinerea & 0.00 & 0.00 & 0.00 & 0.00 & 0.00 & 0.00 & 0.02 & 0.15 & 0.00 & 0.00 & 0.00 & 0.00 \\
\hline Motacilla alba & 0.29 & 0.64 & 0.02 & 0.27 & 0.47 & 0.03 & 0.07 & 0.45 & 0.01 & 0.03 & 0.17 & 0.00 \\
\hline Troglodytes troglodytes & 0.05 & 0.22 & 0.00 & 0.18 & 0.60 & 0.02 & 0.20 & 0.40 & 0.02 & 0.00 & 0.00 & 0.00 \\
\hline Erithacus rubecula & 0.38 & 0.74 & 0.03 & 0.73 & 0.90 & 0.08 & 0.98 & 0.78 & 0.09 & 0.24 & 0.61 & 0.03 \\
\hline Luscinia megarhynchos & 0.24 & 0.44 & 0.02 & 0.00 & 0.00 & 0.00 & 0.04 & 0.21 & 0.00 & 0.00 & 0.00 & 0.00 \\
\hline Phoenicurus ochruros & 0.29 & 0.46 & 0.02 & 0.00 & 0.00 & 0.00 & 0.04 & 0.21 & 0.00 & 0.00 & 0.00 & 0.00 \\
\hline Phoenicurus phoenicurus & 0.52 & 0.68 & 0.04 & 0.36 & 0.81 & 0.04 & 0.07 & 0.25 & 0.01 & 0.06 & 0.35 & 0.01 \\
\hline Saxicola torquatus & 0.14 & 0.48 & 0.01 & 0.00 & 0.00 & 0.00 & 0.00 & 0.00 & 0.00 & 0.18 & 0.39 & 0.02 \\
\hline Turdus merula & 1.43 & 0.81 & 0.10 & 1.00 & 0.63 & 0.11 & 0.93 & 0.72 & 0.09 & 0.52 & 0.67 & 0.05 \\
\hline Turdus philomelos & 0.10 & 0.30 & 0.01 & 0.00 & 0.00 & 0.00 & 0.31 & 0.51 & 0.03 & 0.03 & 0.17 & 0.00 \\
\hline Turdus viscivorus & 0.00 & 0.00 & 0.00 & 0.00 & 0.00 & 0.00 & 0.07 & 0.25 & 0.01 & 0.09 & 0.29 & 0.01 \\
\hline Sylvia subalpina & 0.19 & 0.40 & 0.01 & 0.00 & 0.00 & 0.00 & 0.02 & 0.15 & 0.00 & 0.06 & 0.24 & 0.01 \\
\hline Sylvia communis & 0.33 & 0.58 & 0.02 & 0.27 & 0.65 & 0.03 & 0.04 & 0.21 & 0.00 & 0.48 & 0.80 & 0.05 \\
\hline Sylvia atricapilla & 1.29 & 0.85 & 0.09 & 1.36 & 1.03 & 0.14 & 1.24 & 0.91 & 0.12 & 0.67 & 0.85 & 0.07 \\
\hline Phylloscopus bonelli & 0.33 & 0.58 & 0.02 & 0.27 & 0.65 & 0.03 & 0.16 & 0.37 & 0.02 & 0.06 & 0.24 & 0.01 \\
\hline Phylloscopus collybita & 0.29 & 0.46 & 0.02 & 0.82 & 0.40 & 0.09 & 0.60 & 0.65 & 0.06 & 0.39 & 0.50 & 0.04 \\
\hline Regulus ignicapilla & 0.10 & 0.30 & 0.01 & 0.00 & 0.00 & 0.00 & 0.07 & 0.25 & 0.01 & 0.03 & 0.17 & 0.00 \\
\hline Muscicapa striata & 0.05 & 0.22 & 0.00 & 0.09 & 0.30 & 0.01 & 0.09 & 0.36 & 0.01 & 0.03 & 0.17 & 0.00 \\
\hline Aegithalos caudatus & 0.24 & 1.09 & 0.02 & & 0.40 & 0.02 & 0.31 & 0.76 & 0.03 & 0.12 & 0.42 & 0.01 \\
\hline Poecile palustris & 0.14 & 0.36 & 0.01 & 0.18 & 0.40 & 0.02 & 0.29 & 0.55 & 0.03 & 0.06 & 0.24 & 0.01 \\
\hline Lophophanes cristatus & 0.05 & 0.22 & 0.00 & 0.00 & 0.00 & 0.00 & 0.16 & 0.42 & 0.02 & 0.03 & 0.17 & 0.00 \\
\hline Periparus ater & 0.14 & 0.36 & 0.01 & 0.18 & 0.60 & 0.02 & 0.20 & 0.50 & 0.02 & 0.06 & 0.24 & 0.01 \\
\hline Cyanistes caeruleus & 0.71 & 0.85 & 0.05 & 0.45 & 0.52 & 0.05 & 0.51 & 0.76 & 0.05 & 0.15 & 0.44 & 0.02 \\
\hline Parus major & 0.81 & 0.60 & 0.05 & 0.73 & 0.65 & 0.08 & 0.62 & 0.65 & 0.06 & 0.21 & 0.42 & 0.02 \\
\hline Sitta europaea & 0.24 & 0.54 & 0.02 & 0.09 & 0.30 & 0.01 & 0.24 & 0.43 & 0.02 & 0.00 & 0.00 & 0.00 \\
\hline Certhia familiaris & 0.05 & 0.22 & 0.00 & 0.00 & 0.00 & 0.00 & 0.22 & 0.47 & 0.02 & 0.03 & 0.17 & 0.00 \\
\hline Lanius collurio & 0.10 & 0.44 & 0.01 & 0.18 & 0.40 & 0.02 & 0.00 & 0.00 & 0.00 & 0.27 & 0.57 & 0.03 \\
\hline Garrulus glandarius & 0.33 & 0.58 & 0.02 & 0.09 & 0.30 & 0.01 & 0.31 & 0.51 & 0.03 & 0.03 & 0.17 & 0.00 \\
\hline Pica pica & 0.10 & 0.30 & 0.01 & 0.00 & 0.00 & 0.00 & 0.04 & 0.30 & 0.00 & 0.00 & 0.00 & 0.00 \\
\hline Corvus cornix & 0.33 & 0.66 & 0.02 & 0.00 & 0.00 & 0.00 & 0.00 & 0.00 & 0.00 & 0.03 & 0.17 & 0.00 \\
\hline Sturnus vulgaris & 0.10 & 0.44 & 0.01 & 0.00 & 0.00 & 0.00 & 0.00 & 0.00 & 0.00 & 0.00 & 0.00 & 0.00 \\
\hline Passer italiae & 0.67 & 1.91 & 0.04 & 0.00 & 0.00 & 0.00 & 0.11 & 0.75 & 0.01 & 0.00 & 0.00 & 0.00 \\
\hline Passer montanus & 0.19 & 0.87 & 0.01 & 0.00 & 0.00 & 0.00 & 0.00 & 0.00 & 0.00 & 0.00 & 0.00 & 0.00 \\
\hline Fringilla coelebs & 0.67 & 0.73 & 0.04 & 0.64 & 0.67 & 0.07 & 1.38 & 0.78 & 0.13 & 0.42 & 0.61 & 0.04 \\
\hline Serinus serinus & 0.62 & 0.92 & 0.04 & 0.00 & 0.00 & 0.00 & 0.04 & 0.30 & 0.00 & 0.09 & 0.38 & 0.01 \\
\hline Carduelis chloris & 0.48 & 1.12 & 0.03 & 0.18 & 0.60 & 0.02 & 0.04 & 0.30 & 0.00 & 0.21 & 0.55 & 0.02 \\
\hline Carduelis carduelis & 0.19 & 0.40 & 0.01 & 0.00 & 0.00 & 0.00 & 0.07 & 0.33 & 0.01 & 0.15 & 0.44 & 0.02 \\
\hline Carduelis cannabina & 0.00 & 0.00 & 0.00 & 0.00 & 0.00 & 0.00 & 0.00 & 0.00 & 0.00 & 0.30 & 0.73 & 0.03 \\
\hline Pyrrhula pyrrhula & 0.00 & 0.00 & 0.00 & 0.09 & 0.30 & 0.01 & 0.09 & 0.36 & 0.01 & 0.00 & 0.00 & 0.00 \\
\hline Emberiza citrinella & 0.00 & 0.00 & 0.00 & 0.09 & 0.30 & 0.01 & 0.02 & 0.15 & 0.00 & 0.12 & 0.33 & 0.01 \\
\hline Emberiza cirlus & 0.38 & 0.67 & 0.03 & 0.18 & 0.40 & 0.02 & 0.04 & 0.21 & 0.00 & 0.21 & 0.42 & 0.02 \\
\hline Emberiza cia & 0.10 & 0.44 & 0.01 & 0.00 & 0.00 & 0.00 & 0.04 & 0.21 & 0.00 & 0.06 & 0.24 & 0.01 \\
\hline Emberiza calandra & 0.00 & 0.00 & 0.00 & 0.00 & 0.00 & 0.00 & 0.00 & 0.00 & 0.00 & 0.09 & 0.38 & 0.01 \\
\hline
\end{tabular}


The agricultural environments have the highest species richness with a total of 50 recorded species and a mean of 10.3 species/point. Three are dominant (Sylvia atricapilla, Parus major, and Turdus merula) and fourteen are sub dominant, of which some species are ecotonal, such as Sylvia communis, Emberiza cirlus, and other typical species found in agrarian environments (i.e. Corvus cornix and Passer domesticus). The heterogeneous agricultural areas show the highest species richness $(S=45)$ and mean richness (mean $\mathrm{S}=12.0$ ) in comparison with non-heterogeneous agricultural areas $(\mathrm{S}=37$; mean $=8.5)$.

In the shrubs, we observed 30 species with a mean of 7.5 species/point. Six are dominant (Sylvia atricapilla, Parus major, Fringilla coelebs, Phylloscopus collybita, Turdus merula, and Erithacus rubecula) and five are sub dominant.

Within the woods, 45 species were recorded with a mean of 7.9 species/point. Six are dominant (Sylvia atricapilla, Parus major, Fringilla coelebs, Phylloscopus collybita, Turdus merula, and Erithacus rubecula) and six are sub dominant (Poecile palustris, Cyanistes caeruleus, Aegithalos caudatus, Sitta europaea, Certhia brachydactyla, and Turdus philomelos). The mixed deciduous forest is the sub-category which showed the highest species richness $(\mathrm{S}=33$ ) and mean richness (mean $\mathrm{S}=9.2$ ), followed by the oak woods ( $\mathrm{S}=28$; mean $\mathrm{S}=8.2)$, beech woods $(\mathrm{S}=23$; mean $\mathrm{S}=7.6)$, reforestation areas $(\mathrm{S}=22 ;$ mean $\mathrm{S}=7.2)$ and the chestnut woods $(S=17$; mean $S=7.5)$.

The prairies have a species richness of 48 species and a mean of 7.2 species/point. Six species are dominant
(Alauda arvensis, Anthus campestris, Sylvia atricapilla, Turdus merula, Anthus trivialis, Sylvia communis) and ten are sub dominant (i.e. Lanius collurio, Carduelis cannabina, Lullula arborea, and Emberiza cirlus). The heterogeneous prairies show the highest species richness $(\mathrm{S}=47)$ and mean richness (mean $\mathrm{S}=11.4$ ) in comparison with non-heterogeneous prairies $(S=27$; mean $=5.0)$.

The community's parameters show that the agricultural environments are the ones with the highest species richness, followed in decreasing order by prairies and forest environments. The highest mean richness is found in the agricultural environments, followed in decreasing order by the forest environments, shrubs, and prairies. As for the diversity, the value of the Shannon index is higher in agricultural environments, followed by prairies, forests, and shrubs (Tab. 4).

As for threatened species, higher percentages are observed in agricultural areas and prairies, particularly in non-heterogeneous prairies, heterogeneous agriculture areas, and heterogeneous prairies. The other environmental categories and sub-categories have lower values, particularly in chestnut woods and reforested areas.

The statistical analysis confirms the majority of the obtained results, particularly regarding the difference between agricultural environments and the other habitats.

The mean richness in species per site (Fig. 2) is significantly different in the four habitats taken into consideration (Kruskal-Wallis test $=16.92 ; \mathrm{P}=0.0007$ ). In particular, the mean richness is significantly higher

Tab. 4 - Breeding bird community parameters in each habitat type and sub-categories: $\mathrm{S}=$ Richness, $\mathrm{Smean}=$ mean Richness per point count, $\mathrm{A}=$ Abundance, $\mathrm{H}=$ Diversity, $\mathrm{J}=$ Evenness, non Pass $=\%$ non-Passeriformes, $\mathrm{N}$ dom. $=$ number of dominant species (see Methods), $\mathrm{Ts}=\%$ Threatened species (see Methods). / Parametri della comunità ornitica nidificante in ogni categoria ambientale e sotto categorie: $\mathrm{S}=$ Ricchezza, Smean $=$ Ricchezza media per punto; $\mathrm{A}=$ Abbondanza, $\mathrm{H}=$ Diversità, $\mathrm{J}=$ Equiripartizione, Non Pass. $=\%$ nonPasserformes, N. dom. $=$ numero di specie dominanti (vedi Metodi), $\mathrm{Ts}=\%$ percentuale specie minacciate (vedi Metodi).

\begin{tabular}{|c|l|r|r|r|r|r|r|r|r|r|}
\hline $\begin{array}{c}\text { Env. } \\
\text { categories }\end{array}$ & \multicolumn{1}{|c|}{ Env. sub-categories } & $\begin{array}{c}\text { Point } \\
\text { counts }\end{array}$ & S & Smean & A & H & J & $\begin{array}{c}\text { Non } \\
\text { Pass. }\end{array}$ & $\begin{array}{c}\text { N } \\
\text { dom. }\end{array}$ & Ts \\
\hline $\begin{array}{c}\text { Agricultural } \\
\text { areas }\end{array}$ & & 21 & 50 & 10.3 & 15.0 & 3.52 & 0.90 & 18.6 & 3 & 16.0 \\
\hline & Heterogeneous agricultural areas & 11 & 45 & 12.0 & 19.4 & 3.44 & 0.90 & 15.5 & 6 & 17.8 \\
\hline & Non-heterogeneous agricultural areas & 10 & 37 & 8.5 & 10.3 & 3.27 & 0.90 & 8.1 & 2 & 10.8 \\
\hline Shrubs & & 11 & 30 & 7.5 & 9.5 & 3.01 & 0.89 & 16.7 & 6 & 13.3 \\
\hline Forest areas & & 45 & 45 & 7.9 & 10.4 & 3.14 & 0.82 & 11.1 & 6 & 8.9 \\
\hline & Chestnut woods & 6 & 17 & 7.5 & 9.3 & 2.54 & 0.89 & 11.8 & 6 & 0.0 \\
\hline & Beech woods & 11 & 23 & 7.6 & 10.2 & 2.73 & 0.87 & 8.7 & 6 & 13.0 \\
\hline & Oak woods & 12 & 28 & 8.2 & 10.8 & 2.93 & 0.88 & 10.7 & 6 & 7.1 \\
\hline & Mixed deciduous woods & 7 & 33 & 9.2 & 13.0 & 3.19 & 0.91 & 12.1 & 6 & 9.1 \\
\hline & Reforested areas & 33 & 48 & 7.2 & 9.6 & 3.28 & 0.85 & 23.2 & 6 & 16.6 \\
\hline Prairies & & 11 & 45 & 11.4 & 14.9 & 3.42 & 0.89 & 17.8 & 4 & 17.8 \\
\hline & Heterogeneous prairies & 22 & 27 & 5.0 & 6.9 & 2.67 & 0.81 & 9.1 & 3 & 27.2 \\
\hline & Non-heterogeneous prairies & & & & & & & & \\
\hline
\end{tabular}




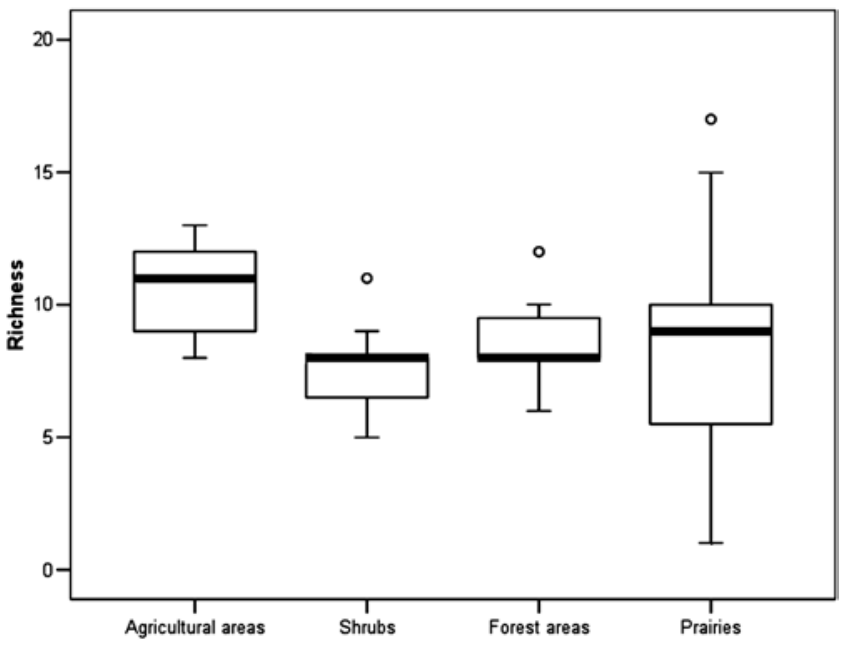

Fig. 2 - Distribution of the number of species/point in each habitat type. The central rectangle spans the first quartile to the third quartile. The segment inside the rectangle shows the median and "whiskers" above and below the box show the locations of the minimum and maximum. Outliers are represented by a circle. / Distribuzione del numero di specie/ punto in ogni categoria ambientale. Il rettangolo centrale si estende dal primo al terzo quartile. Il segmento all'interno del rettangolo mostra la mediana mentre i "baffi" sopra e sotto il rettangolo mostrano le posizioni del minimo e del massimo. I valori anomali sono rappresentati da un cerchio.

in agricultural areas compared to shrub areas (Dunn test $=32.96 ; \mathrm{P}<0.05)$, forests $($ Dunn test $=28.78 ; \mathrm{P}<0.05)$, and open areas (Dunn test $=34,73 ; \mathrm{P}<0,05)$. Conversely, the mean richness is not significant for the other habitats. As for the forest habitats' sub-categories, the mean richness is not significantly different (Kruskal-Wallis test $=3.041 ; \mathrm{P}=0.55$ ), even if the mixed deciduous forest showed a higher species richness, followed by oak woods and beech woods.

For open areas, the mean richness is significantly higher in those that are heterogeneous (Kruskal-Wallis test $=40.56 ; \mathrm{P}<0.0001$ ), both in heterogeneous agricultural areas (Dunn test $=18.87 ; \mathrm{P}<0.05$ ) and in heterogeneous prairies (Dunn test $=27.18 ; \mathrm{P}<0.05$ ).

The mean abundance (Fig. 3) is significantly different in the four macro habitats which have been taken into consideration (Kruskal-Wallis test $=15.59$; $\mathrm{P}=0.0014$ ). In particular, the mean abundance is significantly higher in agricultural environments compared to shrubs (Dunn test $=32.92 ; \mathrm{P}<0.05$ ), forests (Dunn test $=25.58$; $\mathrm{p}<0.05$ ), and open spaces (Dunn test $=31.92 ; \mathrm{P}<0.05$ ), however it is not significant in the other habitats.

The values of dominance don't appear to be significantly different in the four habitats taken into consideration (Friedman test $=4.74 ; \mathrm{P}=0.19$ ), as well as in the other habitats. Also, the percentage of non passerines is not significantly different in the four macro environmental types $\left(\mathrm{X}^{2}=0.233 ; \mathrm{P}=0.62\right)$.

\section{DISCUSSION AND CONCLUSIONS}

The obtained results highlight how the nesting avifauna of the study area, which corresponds to $88 \%$ of the

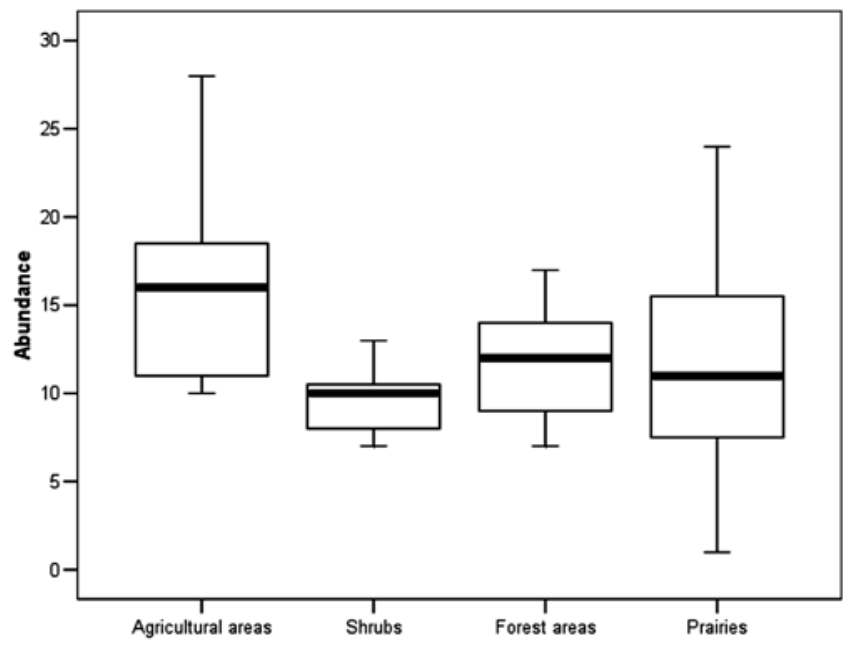

Fig. 3 - Distribution of abundance/point in each habitat type. The central rectangle spans the first quartile to the third quartile. The segment inside the rectangle shows the median and "whiskers" above and below the box show the locations of the minimum and maximum. Outliers are represented by a circle. / Distribuzione dell'abbondanza/punto in ogni categoria ambientale. Il rettangolo centrale si estende dal primo al terzo quartile. Il segmento all'interno del rettangolo mostra la mediana mentre i "baffi" sopra e sotto il rettangolo mostrano le posizioni del minimo e del massimo. I valori anomali sono rappresentati da un cerchio.

known avifauna of Val Borbera (Silvano et al., 1988), is characterized by values of species richness and diversity which can be compared to other areas within the central - northern Apennines with similar environmental characteristics (Toffoli et al., 2003; Vuerich et al., 2006; Nicosia et al., Brunelli et al., 2015). This species richness is due to the high level of the environmental heterogeneity and the conservation state of the habitats, even if these habitats are subjected to rapid transformations due to the abandonment of some agricultural, forestry, and pastoral practices (Assini et al., 2014). This phenomenon influences the surveyed ornithological communities, where the more widespread and dominant species are generalist and linked to the evolving forest and shrub-like vegetation.

The agricultural environments have the highest species richness and diversity, in accordance with what has been observed in other areas of the Apennines (Toffoli et al., 2003; Brunelli et al., 2015). This aspect has to be correlated with the high heterogeneity of this kind of environment in which the high margin effect, which derives from the coexistence of patches of different habitats, often small is size, allows for the presence of a considerable number of species linked to shrubs, forests, and other typical vegetation from agricultural systems and open spaces. This effect can also be seen in the prairies, which are second in species richness and diversity to the open spaces. Even though this habitat is found in the study area with a certain continuity and surface area, it is limited to the ridge and shows an elevated margin effect due to the presence of forests, which delimit its entire perimeter. This effect determines the coexistence of typical prairie species and forest species in numerous listening sites. The high species richness found in open 
spaces, caused by the margin effect, has been reported in other similar studies conducted in mountain and hill areas (Sorace, 1996; Giraudo, 2001; Farina, 2003; Lorenzetti et al., 2004; Sorace, 2011). In the absence of environmental heterogeneity and marginal effects, the ornithological communities of open spaces - prairies, pastures - are usually characterized by a limited number of species and of individuals with low values of diversity (Sorace, 1996; Vuerich et al., 2006). In spite of this fact, prairies host an avifauna rich in specificity and of interesting elements (Blondel \& Farré, 1988) that, at the moment, are in a phase of rapid decline (Burfield \& Van Bommel, 2004), as shown by the non-heterogeneous prairies hosting a large percentage of threatened species in this study. Prairies and agricultural environments are, in fact, the two types of environments in which the highest number of rare species at a regional scale have been observed, such as the Alectoris rufa, Anthus campestris, Lullula arborea (Mingozzi et al., 1988; Aimassi \& Reteuna, 2007; Tizzani et al., 2013) or with negative trends at a national level, such as Alauda arvensis, Anthus trivialis, Sylvia communis, and Lanius collurio (Campedelli et al., 2012) and those considered threatened in the red lists (Peronace et al., 2012).

The environments with evolving shrubs and forest vegetation are poor in species richness and have a low diversity, especially for the shrubs. The expansion of invading shrubs, which derive from the abandonment of the traditional types of agricultural and zootechnical exploitation, represent one of the causes of the decline of biodiversity in mountain areas and of the reduction in the populations of some bird species in open spaces (Faralli, 1995; Preiss et al., 1997). Many of these species, such as the skylark, tend to avoid the forest edges and closed spaces which derive from the growth of shrubs (Sposimo $\&$ Tellini, 1988). In these types of environments species are mostly found widespread at a local and national scale with generally positive trends (Campedelli et al., 2012). Even though the point counts covered a sufficiently wide surface area and ideal habitats (chestnut and beech woods), it has not been possible to confirm the presence of the small nesting population of Ficedula albicollis in the reproductive period (Silvano et al., 1988). It is believed that the species has disappeared from this sector of the northern Apennines, in correlation with the negative trend of the population at the edges of the Italian distribution range of the species (Bricchetti \& Fracasso, 2008).

\section{REFERENCES}

Aimassi G. \& Reteuna D., 2007 - Uccelli nidificanti in Piemonte e Valle d'Aosta: aggiornamento della distribuzione di 120 specie. Memorie Associazione Naturalistica Piemontese VII.

Assini S., Filipponi F. \& Zucca F., 2015 - Land cover changes in an abandoned agricultural land in the Northern Apennine (Italy) between 1954 and 2008: Spatio-temporal dynamics. Plant Biosystems-An International Journal Dealing with all Aspects of Plant Biology, 149 (5): 807-817.
Bibby C.J., Burgess N.D., Hill D.A. \& Mustoe S.H., 2000 - Bird Census Techniques. II ed. Academic Press.

Blondel J. \& Farré H., 1988 - The convergent trajectories of bird communities in European forests. Oecologia, 75 (1): 83-93.

Brichetti P. \& Fracasso G., 2008 - Ornitologia Italiana. Vol. 5. Turdidae-Cisticolidae. Oasi, Alberto Perdisa Editore.

Brunelli M., Cento M., De Santis E., Savo E. \& Sorace A., 2015 - Breeding bird community of the Montagne della Duchessa (Latium, central Italy). Avocetta, 39: 19-28.

Burfield I. \& Van Bommel F. (eds.), 2004 - Birds in Europe. Population estimates, trends and conservationstatus. BirdLife Conservation Series, no. 12. BirdLife International.

Campedelli T., Buvoli L., Bonazzi P., Calabrese L., Calvi G., Celada C., Cutini S., De Carli E., Fornasari L., Fulco E., La Gioia G., Londi G., Rossi P., Silva L. \& Tellini Florenzano G., 2012 - Andamenti delle popolazioni delle specie comuni nidificanti in Italia: 20002011. Avocetta, 36: 121-143.

Faralli U., 1995 - Effetti della riforestazione sulle comunità ornitiche di una brughiera dell'Appennino Settentrionale, Toscana. In: Atti del VII Convegno Nazionale di Ornitologia. Pandolfi M. \& Foschi U.F. (eds.). Supplemento Ricerche di Biologia della Selvaggina, XXII: 299-306.

Farina A., 2003 - Ecologia del paesaggio. Principi, metodi e applicazioni. UTET Libreria, Pescara.

Ferry C. \& Frochot B., 1970 - L'avifaune nidificatrice d'une forêt de chênes pédonculés en Bourgogne: étude de deux successions écologiques. Terre et Vie, 2: 153250.

Fornasari L., Londi G., Buvoli L., Florenzano G. T., La Gioia G., Pedrini P., Brichetti P. \& De Carli E. (eds.), 2010 - Distribuzione geografca e ambientale degli uccelli comuni nidifcanti in Italia, 2000-2004 (dati del progetto MITO2000). Avocetta, 34: 5-15.

Giraudo L., 2001 - Relazione fra fattori ambientali, stagionali e avifauna del piano montano. Avocetta, 25: 54.

Hammer Ø., Harper D.A.T. \& Ryan P.D., 2001 - PAST: Paleontological Statistics Software Package for Education and Data Analysis. Palaeontologia Electronica, 4: $1-9$.

Lloyd M. \& Ghelardi R., 1964 - A table for calculating the "Equitability" component of species diversity. Journal Animal Ecology, 33: 217-225.

Lorenzetti E., Ukmar E. \& Battisti C., 2004 - Comunità ornitiche nidificanti nel Sito di Importanza Comunitaria "Monte Guadagnolo" (Monti Prenestini, Italia centrale). Alula, 11: 105-112.

Mingozzi T., Boano G. \& Pulcher C., 1988 - Atlante degli uccelli nidificanti in Piemonte e Val d'Aosta, 19801984. Monografia VIII. Museo Regionale di Scienze Naturali di Torino.

Nicosia E., Aluigi A., Fasano S. \& Toffoli R., 2009 - La Rete Natura 2000 in Liguria: caratterizzazione e confronto di alcune realtà. Atti del XV Convegno Italiano 
di Ornitologia. Parco Nazionale del Circeo, Sabaudia (Latina) 14-18 ottobre 2009. Alula, XVI (1-2): 558560.

Peronace V., Cecere J., Gustin M. \& Rondinini C., 2012Lista Rossa 2011 degli uccelli nidificanti in Italia. Avocetta, 36 (1): 11-58.

Pielou E.C., 1966 - The measurement of diversity in different types of biological collections. Journal Theoretical Biology, 13: 131-144.

Preiss E.J., Martin L. \& Debussche M., 1997 - Rural depopulation and recent landscape changes in a Mediterranean region: consequences to the breeding avifauna. Landscape Ecology, 12 (1): 51-61.

Regione Piemonte, 2009 - Piano Forestale Territoriale. Regione Piemonte, Settore Foreste.

Shannon C.E. \& Weaver W., 1963 - Mathematical theory of communication. University of Illinois Press.

Silvano F., Carrega M., Torreggiani F., 1988 - L'avifauna della Val Borbera (AL). Rivista Piemontese di Storia Naturale, IX: 173-188.

Sorace A., 1996 - Alcuni dati sulle comunità ornitiche del comprensorio Monte Cairo Valle del Melfa (FR). Alula, 3: 17-22.

Sorace A., 2011 - Le comunità ornitiche degli ambienti aperti della Sila Grande (Calabria) con note sull'apparente contrazione demografica di Saxicola rubetra, Regulus regulus e Carduelis spinus. Avocetta, 35: 5360.

Sposimo P. \& Tellini G., 1988 - Separazione spaziale fra Allodola Alauda arvensis e Tottavilla Lullula arborea nell'Appennino Settentrionale. In: Atti IV Convegno Italiano di Ornitologia. Massa B. (ed.). Naturalista Siciliano, XII (Suppl.): 299-303.

Tizzani P., Boano G., Mosso M., Pelazza M., Carolfi S., Ferra M., Marletta N., Pio G., Pellegrino I., Meneguz P.G., Silvano F., Negri N. \& Spanò S., 2013 - Recent distribution of red-legged partridge Alectoris rufa in Piedmont (North Western Italy): signs of recent spreading. Avocetta, 37: 83-86

Toffoli R., Carpegna F., Fasano S. \& Panizza G., 2003 - La comunità ornitica nidificante nel Parco Naturale delle Capanne di Marcarolo (AL). Avocetta, 27: 125125.

Turcek F. J., 1956 - Zur Frage der Dominanze in Vogelpopulationen. Waldhygiene, 8: 249-257.

Vuerich V., Bologna M. A. \& Battisti C., 2006 - Comunità ornitiche nidificanti in tre tipologie vegetazionali lungo un gradiente altitudinale nei monti Simbruini (Lazio, Appennini centrali) (Aves). Aldrovandia, 2: 49-53. 